\title{
Combustion for Net Zero Carbon Society
}

\author{
Dr. Y. Hardalupas \\ Imperial College London, Mechanical Engineering Department \\ London SW7 2AZ, UK
}

\begin{abstract}
Government policies worldwide are focusing on delivering net zero carbon emissions by 2050, although associated timescales may vary. As a consequence of such policies, a common suggestion is that combustion cannot contribute to a future zero-carbon world. The talk will present a range of combustion processes that can contribute to the energy needs of a future net zero carbon society. The combustion processes for different applications, including power generation, air, sea and land transport, will be described and advantages and disadvantages will be identified. Examples of current research will be provided to demonstrate the scientific and technological challenges that research must address in order to deliver the net zero carbon combustion technologies. The presentation will end with suggestions for future research needs.
\end{abstract}

